\title{
The ACR11 encodes a novel type of chloroplastic ACT domain repeat protein that is coordinately expressed with GLN2 in Arabidopsis
}

\author{
Tzu-Ying Sung ${ }^{\dagger},{\text { Tsui-Yun } \text { Chung }^{\dagger} \text {, Chih-Ping Hsu and Ming-Hsiun Hsieh }}^{*}$
}

\begin{abstract}
Background: The ACT domain, named after bacterial aspartate kinase, chorismate mutase and TyrA (prephenate dehydrogenase), is a regulatory domain that serves as an amino acid-binding site in feedback-regulated amino acid metabolic enzymes. We have previously identified a novel type of ACT domain-containing protein family, the ACT domain repeat (ACR) protein family, in Arabidopsis. Members of the ACR family, ACR1 to ACR8, contain four copies of the A'CT domain that extend throughout the entire polypeptide. Here, we describe the identification of four novel ACT domain-containing proteins, namely ACR9 to ACR12, in Arabidopsis. The ACR9 and ACR10 proteins contain three copies of the ACT domain, whereas the ACR11 and ACR12 proteins have a putative transit peptide followed by two copies of the ACT domain. The functions of these plant ACR proteins are largely unknown.

Results: The ACR11 and ACR12 proteins are predicted to target to chloroplasts. We used protoplast transient expression assay to demonstrate that the Arabidopsis ACR11- and ACR12-green fluorescent fusion proteins are localized to the chloroplast. Analysis of an ACR11 promoter- $\beta$-glucuronidase (GUS) fusion in transgenic Arabidopsis revealed that the GUS activity was mainly detected in mature leaves and sepals. Interestingly, coexpression analysis revealed that the GLN2, which encodes a chloroplastic glutamine synthetase, has the highest mutual rank in the coexpressed gene network connected to ACR11. We used RNA gel blot analysis to confirm that the expression pattern of ACR11 is similar to that of GLN2 in various organs from 6-week-old Arabidopsis. Moreover, the expression of ACR11 and GLN2 is highly co-regulated by sucrose and light/dark treatments in 2-week-old Arabidopsis seedlings.

Conclusions: This study reports the identification of four novel ACT domain repeat proteins, ACR9 to ACR12, in Arabidopsis. The ACR11 and ACR12 proteins are localized to the chloroplast, and the expression of ACR11 and GLN2 is highly coordinated. These results suggest that the ACR11 and GLN2 genes may belong to the same functional module. The Arabidopsis ACR11 protein may function as a regulatory protein that is related to glutamine metabolism or signaling in the chloroplast.
\end{abstract}

\section{Background}

Nitrogen is one of the most important nutrients for plant growth and development. Plants can utilize different forms of nitrogen including nitrate, ammonium, and amino acids. Most plants use inorganic nitrogen nitrate as the primary nitrogen source. Nitrate taken up from the soil will be reduced to ammonium by nitrate reductase and nitrite reductase. Ammonium derived from nitrate or remobilized from the other nitrogen-

\footnotetext{
* Correspondence: ming@gate.sinica.edu.tw

† Contributed equally

Institute of Plant and Microbial Biology, Academia Sinica, Taipei 11529,
} Taiwan

(ㄷ) 2011 Sung et al; licensee BioMed Central Ltd. This is an Open Access article distributed under the terms of the Creative Commons Attribution License (http://creativecommons.org/licenses/by/2.0), which permits unrestricted use, distribution, and reproduction in any medium, provided the original work is properly cited. containing compounds can be assimilated into glutamine and glutamate via the glutamine synthetase (GS)/ glutamine-oxoglutarate aminotransferase (GOGAT) cycle. Glutamine and glutamate are the major amino donors for the synthesis of the other amino acids and nitrogen-containing compounds in plants [1]. In addition to their roles in protein synthesis and metabolism, glutamine and glutamate may also serve as signaling molecules in plants [2-6].

The synthesis of glutamine and glutamate also depends on the availability of $\alpha$-ketoglutarate. In bacteria, the carbon skeleton of ammonia assimilation, $\alpha$ ketoglutarate, signals nitrogen deficiency, whereas

\section{Biomed Central}


glutamine, the fully aminated product, often signals nitrogen sufficiency [7]. In E. coli, the expression of glutamine synthetase gene and its enzyme activity are regulated by the availability of glutamine and $\alpha$-ketoglutarate [7-10]. In response to low glutamine/ $\alpha$-ketoglutarate, the $E$. coli PII protein (encoded by $g \ln B$ ) is uridylylated by $\mathrm{G} \operatorname{lnD}$, an uridylyltransferase/uridylyl-removing enzyme [11,12]. The uridylylated PII interacts with an adenylyltransferase to deadenylylate and activate the GS enzyme (encoded by $g \ln A$ ) $[11,13]$. In addition, the $\mathrm{NtrB} / \mathrm{NtrC}$ two-component system will activate the expression of $g \ln A$ under nitrogen-limiting conditions [9,14-19]. By contrast, in response to high glutamine/ $\alpha$ ketoglutarate, the uridylylated PII is deuridylylated by GlnD. The unmodified PII protein interacts with adenylyltransferase thereby causing the adenylylation and inactivation of the GS enzyme [11,12]. The unmodified PII protein also interacts with the NtrB/NtrC two-component system to inactivate the expression of $g \ln A$ [9,14-19]. Thus bacterial PII proteins are sensors of $\alpha$ ketoglutarate and adenylate energy charge, whereas $\mathrm{GlnD}$ is the sensor of glutamine $[20,21]$.

Little is known about amino acid sensing and signaling in plants. PII-like proteins have been identified in Arabidopsis and rice [22,23]. However, bacterial GlnD homologs have yet to be identified in plants. The E. coli sensor protein $\mathrm{G} \ln \mathrm{D}$ is composed of a nucleotide transferase domain, a nucleotide hydrolase domain, and two $\mathrm{C}$-terminal ACT domains. It has been shown that the C-terminal ACT domains of GlnD may regulate its activity through the binding of glutamine [21].

The ACT domain, named after bacterial aspartate kinase, chorismate mutase and TyrA (prephenate dehydrogenase), is a regulatory domain that serves as an amino acid-binding site in feedback-regulated amino acid metabolic enzymes [24-28]. For instance, the E. coli 3-phosphoglycerate dehydrogenase (PGDH), a key enzyme in serine biosynthesis, is feedback regulated by serine. The C-terminal ACT domain of E. coli PGDH is the binding site for its allosteric effector serine $[24,29,30]$. The other amino acid metabolic enzymes such as acetohydroxyacid synthase [31], threonine deaminase [32,33], and phenylalanine hydroxylase [34] also contain the regulatory ACT domain. In addition, the ACT domain is also found in several transcription factors [35-39].

We previously identified a novel type of ACT domaincontaining protein family in Arabidopsis, whose members contain four $\mathrm{ACT}$ domain repeats (the "ACR" protein family) [40]. Other than the ACT domain, the amino acid sequences of the ACR proteins do not have homology to any known enzymes or motifs in the database (http://www.ebi.ac.uk/Tools/InterProScan/). Although proteins homologous to the ACR family have been identified in rice [41-43], the functions of these ACR proteins are largely unknown.

In this report, we have identified four additional ACT domain-containing proteins in Arabidopsis. These proteins are composed of three or two copies of the ACT domain. The amino acid sequences of these proteins do not have any recognizable motifs except the ACT domain. These novel ACT domain-containing proteins are classified as new members of the ACR family. We showed that the newly identified ACR11 and ACR12 proteins are localized to the chloroplast. Interestingly, the expression of ACR11 is co-regulated with GLN2 that encodes a chloroplastic glutamine synthetase (GS). The possible functions of Arabidopsis ACR11 are discussed herein.

\section{Results}

Identification of four novel ACR genes in Arabidopsis

We previously used the ACT domain (Pfam01842) and bacterial GlnD sequences to identify Arabidopsis ACR1 to ACR8 proteins, which contain four copies of the ACT domain [40]. In addition to these ACR proteins, we have identified four novel ACT domain-containing proteins encoded by At1g16880, At2g36840, At2g39570 and At5g04740, which contain two or three copies of the ACT domain. Since these proteins also contain ACT domain repeats, we propose to classify these proteins as new members of the ACR family. We named the proteins encoded by At2g39570, At2g36840, At1g16880 and At5g04740 genes ACR9, ACR10, ACR11 and ACR12, respectively. According to amino acid sequence alignment and phylogenetic analysis, ACR1 to ACR12 proteins are divided into three groups (Figure 1A). The originally identified ACR1 to ACR8 proteins belong to Group I. The newly identified ACR9 to ACR12 belong to Group II (ACR9 and ACR10) and Group III (ACR11 and ACR12), respectively (Figure $1 \mathrm{~A}$ ).

$A C R 9$ and $A C R 10$ have almost identical gene structures with respect to size and arrangement of their exons and introns (Figure 1B). By contrast, ACR11 and ACR12 have the same numbers of exon and intron, but some of the introns are different in size (Figure 1B). We used the computer program InterProScan (http://www.ebi.ac.uk/ Tools/InterProScan/) to analyze domain compositions of ACR9 to ACR12. The ACR9 and ACR10 proteins contain three copies of the ACT domain, whereas the ACR11 and ACR12 proteins contain two copies of the ACT domain (Figure 1C). Similar to the ACR1 to ACR8 proteins, the ACR9 to ACR12 proteins do not have other known domains or motifs as revealed by InterProScan.

\section{Sequence analysis of Arabidopsis ACR11 and ACR12}

According to the sequences in the GenBank, we designed specific primers and used RT-PCR to amplify 


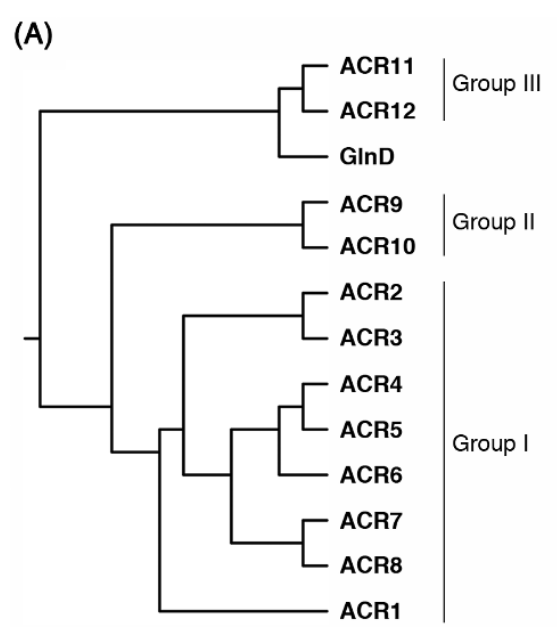

(B)

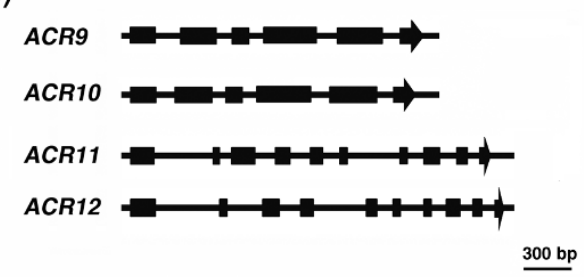

(C)

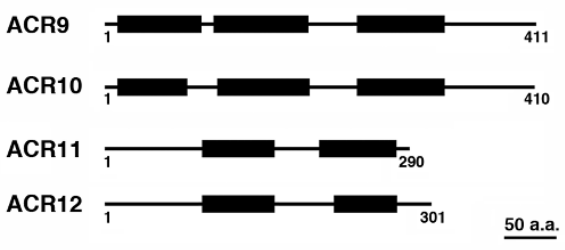

Figure 1 Sequence analysis of the Arabidopsis ACR family. (A) Phylogenetic relationships of Arabidopsis ACR proteins and the C-terminal ACT domains of E. coli GlnD. Full-length amino acid sequences of Arabidopsis ACR1 to ACR12 and amino acid residues 708-890 of E. coli GlnD were aligned by ClustalW2 and the neighbor-joining algorithm was used to obtain the phylogenetic tree. (B) Schematic gene structures of Arabidopsis ACR9 to ACR12. Exons are shown as black boxes and introns are indicated as solid lines. (C) Schematic diagram of Arabidopsis ACR9 to ACR12 proteins. The black boxes indicate the ACT domains.

full-length cDNAs of $A C R 11$ and ACR12. The ACR11 and ACR12 proteins have 290 and 301 amino acid residues, respectively. Amino acid sequence alignment of ACR11 and ACR12 shows that the N-terminal regions of these two proteins are not highly conserved. Beyond the $\mathrm{N}$-terminal regions, the amino acid sequences in ACR11 (residues 74 to 290) and ACR12 (residues 85 to 301 ), share $63 \%$ identity and $82 \%$ similarity (Figure $2 \mathrm{~A}$ ). Several computer programs including PSORT (http:// www.psort.org/) and TargetP (http://www.cbs.dtu.dk/ services/TargetP/) predicted that the ACR11 and ACR12 proteins are localized to the chloroplast. Most nuclearencoded chloroplast proteins contain $\mathrm{N}$-terminal transit peptide sequences that facilitate the transfer of these proteins from the cytoplasm to the chloroplast. The transit peptides will be cleaved after the precursor proteins are imported into chloroplasts. In ACR11 and ACR12, the less conserved $\mathrm{N}$-terminal sequences may function as transit peptides to target these proteins to the chloroplast. Indeed, the computer program ChloroP (http://www.cbs.dtu.dk/services/ChloroP/) predicts the presence of transit peptides in both proteins, and the

\section{(A)}

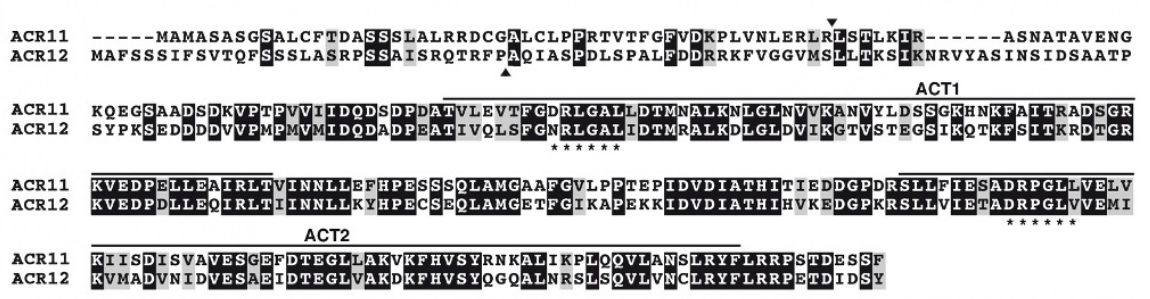

(B)

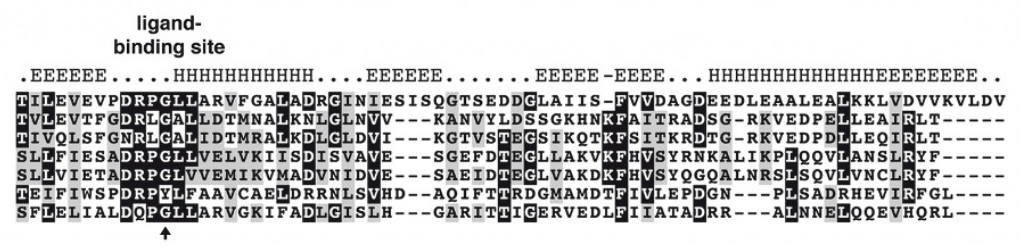

Figure 2 Amino acid sequence alignments of ACR proteins and ACT domains. (A) Sequence alignment of Arabidopsis ACR11 and ACR12 proteins. ACT domains are indicated with solid lines above the sequences. Arrowheads indicate the predicted cleavage sites of chloroplast transit peptides. Asterisks shown below the sequences denote the putative ligand-binding sites. (B) Sequence alignment of ACT consensus sequence (ACTC) from Pfam01842, and ACT domains from ACR11 (ACR11.1 and ACR11.2), ACR12 (ACR12.1 and ACR12.2) and GlnD (GlnD1 and $\mathrm{Gln} D 2$ ). The predicted secondary structure of the ACTc is shown above the sequences. Arrow indicates the conserved glycine residue in the $\beta 1-$ $\alpha 1$ loop region. Identical and similar amino acid residues are shaded in black and gray, respectively. 
locations of potential transit peptide cleavage sites are between the 52Arg-53Leu of ACR11, and the 32Pro33Ala of ACR12, respectively (Figure 2A).

Protein BLAST analyses revealed that ACR11 and ACR12 are most similar to the ACT domains of bacterial PII-uridylyltransferase (GlnD) in addition to their homologs in photosynthetic organisms (data not shown). We aligned the ACT domains from Arabidopsis ACR11 and ACR12 with the two ACT domains from E. coli GlnD and the ACT consensus sequence from Pfam01842. The structure of the ACT consensus sequence is predicted to form a $\beta \alpha \beta \beta \alpha \beta$ fold, which is in accordance with the archetypical structure of the ACT domain of E. coli PGDH [24]. In addition, the initial identification and alignment of ACT domains uncovered a nearly invariant Gly residue at the turn between the first $\beta$ strand and the first $\alpha$ helix that coincided with the binding site for Ser in E. coli PGDH [25]. The alignment of ACT domains from ACR11, ACR12 and GlnD indicated that these sequences are highly conserved in the $\beta 1-\alpha 1$ loop region (Figure $2 \mathrm{~B}$ ). Moreover, the invariant Gly residue is also present in the ACT domains of Arabidopsis ACR11 and ACR12 (Figure 2B).

\section{The ACR11- and ACR12-GFP are localized to the chloroplast}

We used green fluorescent fusion protein (GFP) and protoplast transient expression assay to examine the subcellular localization of ACR11 and ACR12. The fulllength ACR11 and the first 94 amino acids of ACR12 were fused to the N-terminus of a GFP. The resulting ACR11- and ACR12-GFP fusion constructs driven by a cauliflower mosaic virus (CaMV) 35S promoter were transformed into Arabidopsis protoplasts. Confocal microscopy was used to observe the fluorescent signals $16 \mathrm{~h}$ after transformation. The green fluorescent signals of ACR11- and ACR12-GFP fusion proteins co-localized with the auto-fluorescent signals of chlorophylls in the chloroplasts (Figure 3). By contrast, the protoplast transformed with the empty GFP vector alone has green fluorescent signals in the cytosol and nucleus (Figure 3). These results suggest that the Arabidopsis ACR11 and ACR12 proteins are localized to the chloroplast.

\section{Coexpression gene networks of Arabidopsis ACR11 and ACR12}

The functions of Arabidopsis ACR11 and ACR12 are completely unknown. It has been suggested that genes involved in related biological pathways are often expressed cooperatively [44]. We attempted to identify the functions of ACR11 and ACR12 by searching for genes that are coexpressed with ACR11 and ACR12, respectively. We obtained the $A C R 11$ and $A C R 12$

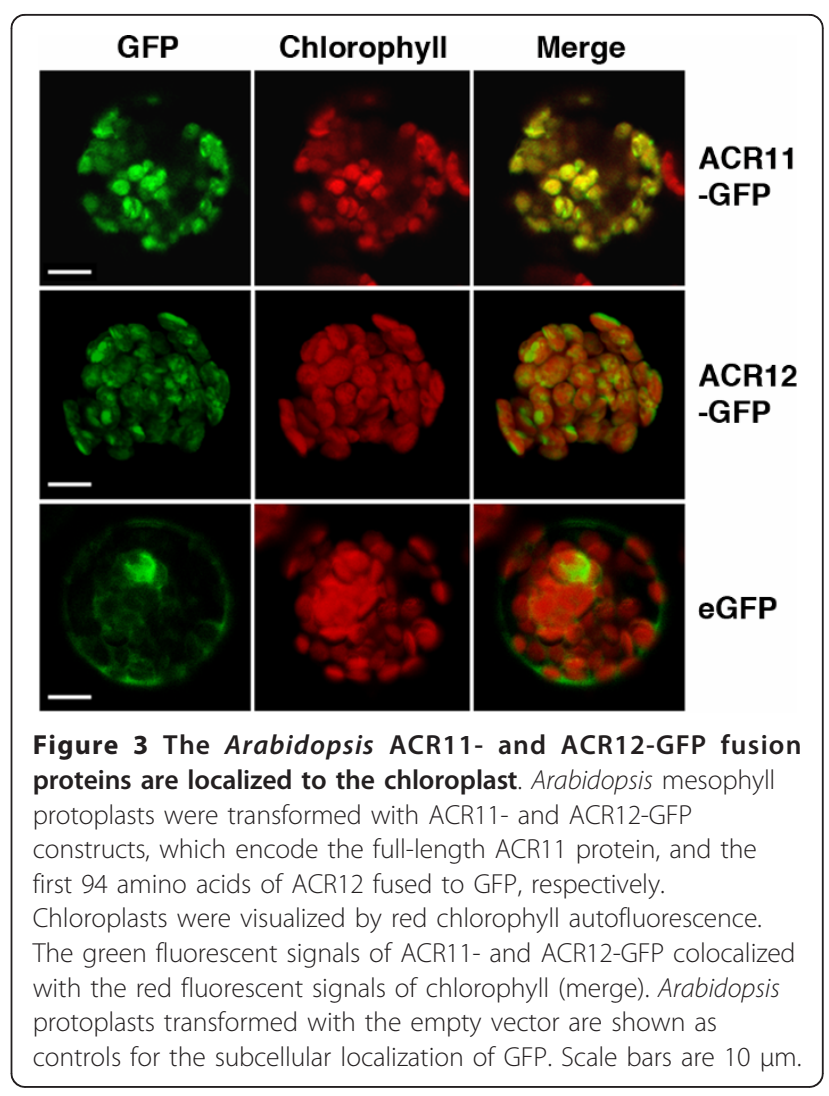

coexpression gene networks from the ATTED-II database (http://atted.jp/) [45]. The three genes having the highest mutual rank (MR) with ACR11 are At5g35630 (GLN2, encodes a chloroplastic glutamine synthetase; $\mathrm{MR}=1.0$ ), At1g15545 (encodes an unknown protein; $\mathrm{MR}=8.5$ ), and At5g64460 (encodes an unknown protein; $M R=9.2$ ) (Figure $4 \mathrm{~A})$. It is intriguing to find that $A C R 11$ and GLN2 have the highest mutual rank of coexpression compared with all other genes in the Arabidopsis genome. By contrast, the top three genes that are coexpressed with ACR12 are At3g29350 (encodes AHP2, histidine-containing phosphotransmitter2; $\mathrm{MR}=2.2$ ), At1g10200 (encodes WLIM1, a member of the Arabidopsis LIM proteins; MR = 6.2), and At1g49820 (encodes MTK1, 5-methylthioribose kinase1; MR = 7.5) (Figure 4B). The expression of $A C R 12$ is not co-ordinately regulated with $A C R 11$ and GLN2 in the ATTEDII database.

\section{The expression of $A C R 11$ and GLN2 is up-regulated by} light and sucrose

We used RNA gel blot analysis to examine the expression patterns of ACR11 and GLN2 in different organs from 6-week-old Arabidopsis plants. Steady-state levels of ACR11 and GLN2 mRNAs are low in roots compared to those of leaves, stems, and flowers (Figure 5). It is 


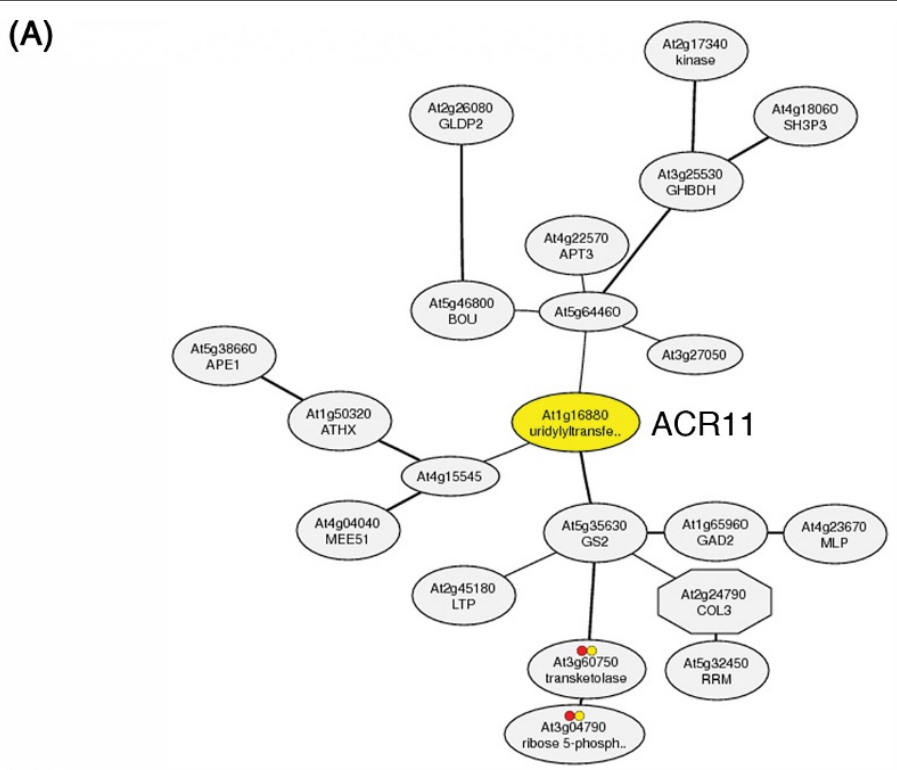

(B)

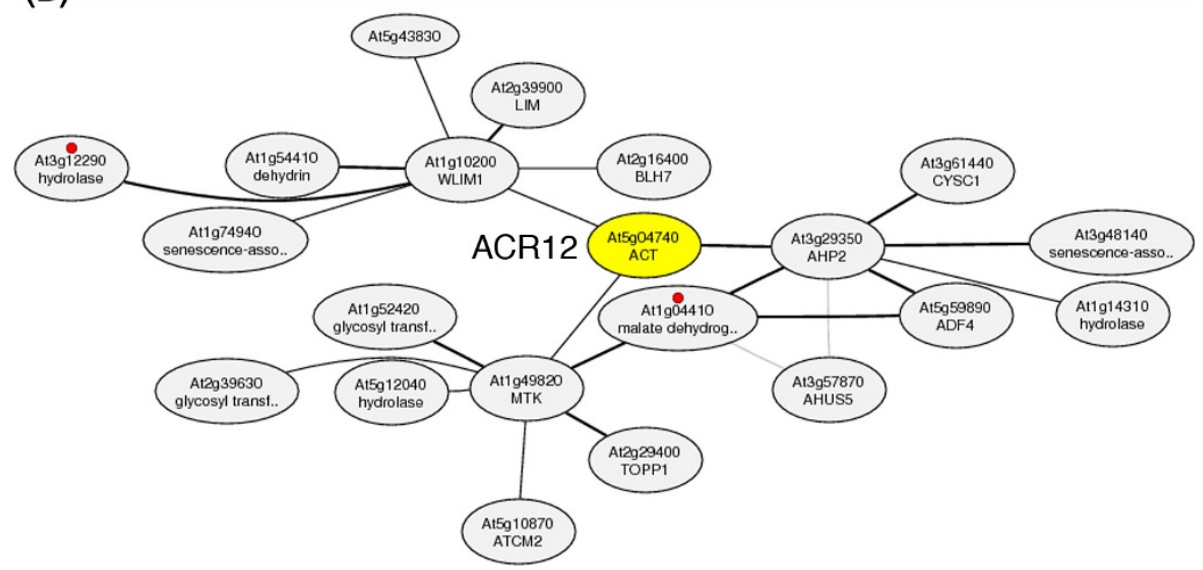

Figure 4 Coexpressed gene networks around Arabidopsis ACR11 and ACR12. (A) The three genes having the highest coexpression mutual rank (MR) with $A C R 11$ are At5g35630 (encoding glutamine synthetase 2; MR = 1.0), At4g15545 (encoding an unknown protein; $M R=8.5$ ) and At5g64460 (encoding an unknown protein; MR = 9.2). The ACR11 (At1g16880) is annotated as an uridylyltransferase-related protein in the database. (B) The three genes having the highest coexpression mutual rank (MR) with ACR12 are At3g29350 (encodes AHP2, histidine-containing phosphotransmitter2; MR = 2.2), At1g10200 (encodes WLIM1, a member of the Arabidopsis LIM proteins; MR = 6.2), and At1g49820 (encodes MTK1, 5-methylthioribose kinase1; MR = 7.5). The coexpression gene networks of ACR11 and ACR12 can be obtained at the ATTED-II website (http://atted.jp/data/locus/At1g16880.shtml and http://atted.jp/data/locus/At5g04740.shtml).

well known that the expression of Arabidopsis GLN2 is regulated by light and sucrose [46]. We used RNA gel blot analysis to examine the effects of light and sucrose on the expression of ACR11 and GLN2 (Figure 6). Two weeks old Arabidopsis seedlings grown on a $16 \mathrm{~h}$ light $/ 8$ $\mathrm{h}$ dark cycle were transferred to media containing $0 \%$ sucrose, $3 \%$ sucrose or $3 \%$ manitol, and dark-adapted or grown in continuous light for $48 \mathrm{~h}$. Total RNA extracted from these samples was used for RNA gel blot analysis. In dark-adapted seedlings, steady-state levels of $A C R 11$ and GLN2 mRNAs are slightly increased by $3 \%$ sucrose treatment. This sucrose effect is not related to an osmotic change, because the addition of $3 \%$ mannitol does not increase the accumulation of ACR11 and GLN2 transcripts. By contrast, steady-state levels of $A C R 11$ and GLN2 mRNAs are significantly increased by the light treatment, regardless of the amounts of sucrose or mannitol in the media. The expression patterns of $A C R 11$ and GLN2 are almost identical under these treatments. These results confirm that the ACR11 and GLN2 genes are expressed cooperatively under various conditions.

\section{ACR11 promoter-GUS activity}

To further examine the cell type and tissue specific expression of the ACR11 gene, we fused the putative 


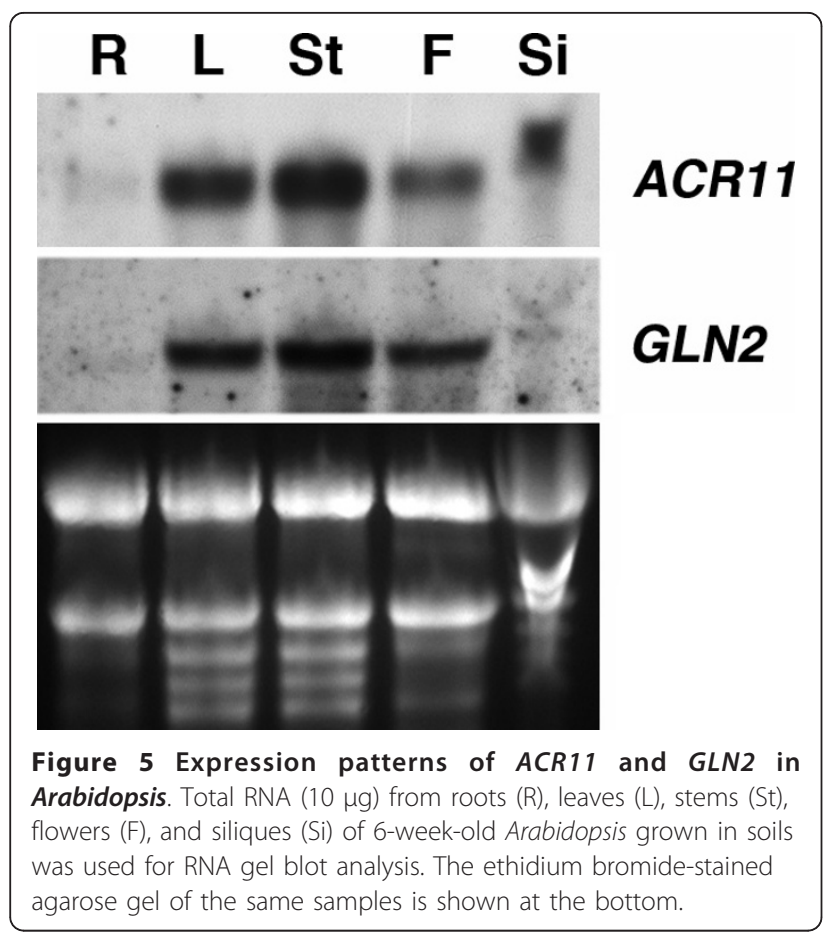

promoter of $A C R 11$ to a $\beta$-glucuronidase reporter gene (ACR11p-GUS) and generated stable Arabidopsis transgenic lines. The ACR11p-GUS activity was detected in the cotyledons of 3-, 5- and 7-day-old seedlings

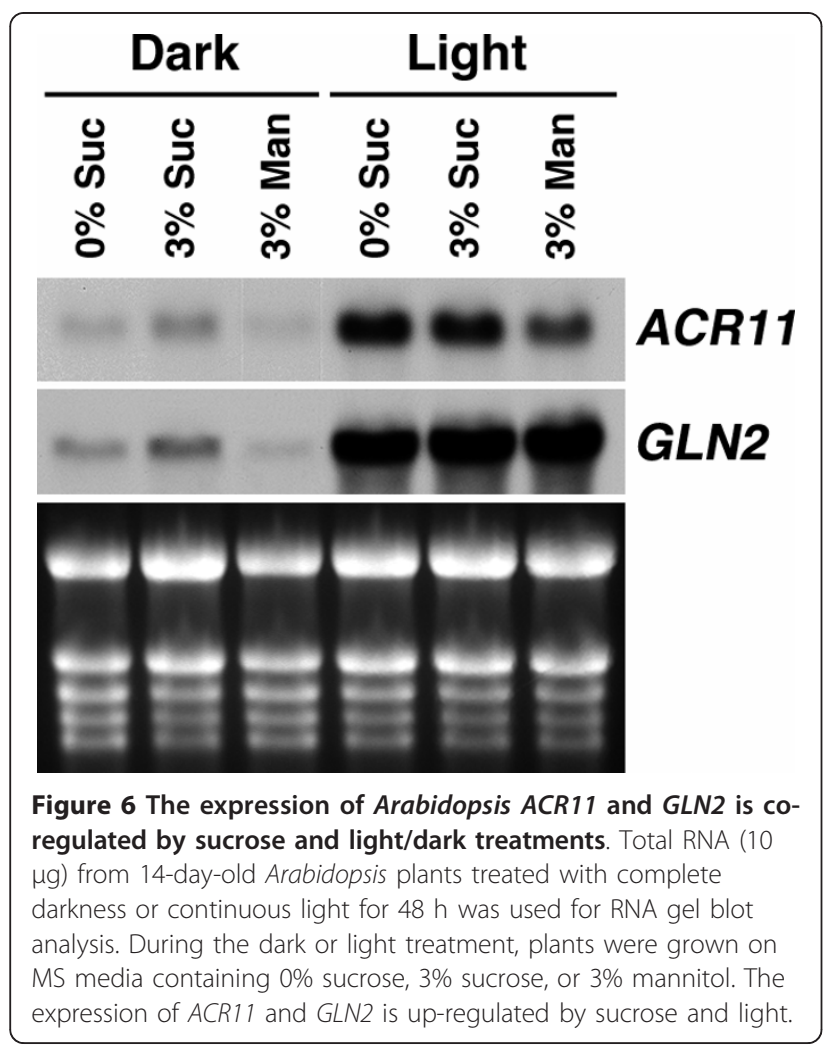

(Figure 7A-C). Interestingly, the ACR11p-GUS activity was not detected in emerging young leaves and the basal part of maturing leaves, which are mainly composed of dividing and growing young cells (Figure 7CE). In developing or mature flowers, the ACR11p-GUS activity was detected in sepals as a gradient from the apical part (high) to the basal part (low) (Figure 7F and 7G). In mature flowers, the ACR11p-GUS activity was also detected in the style (Figure 7G). In mature siliques, the ACR11p-GUS activity was detected in the tip of the pedicel (Figure $7 \mathrm{H}$ ).

\section{Discussion}

Three distinct groups of ACR proteins in Arabidopsis

We previously reported the identification and characterization of eight ACT domain repeat proteins in Arabidopsis and named these proteins ACR1 to ACR8, respectively [40]. These ACR proteins each contain four copies of the ACT domain. Here, we describe four additional ACT domain-containing proteins in Arabidopsis. Except in the regions of the ACT domain, the amino acid sequences of these novel ACT domain-containing proteins are not similar to the originally identified ACR proteins. However,
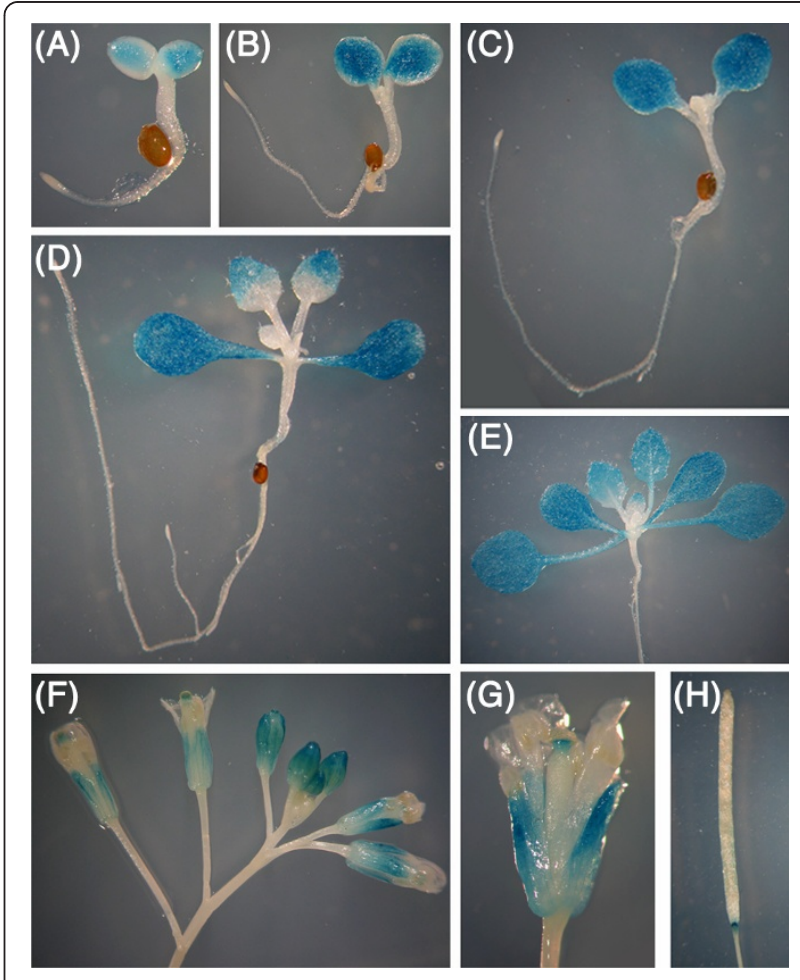

Figure 7 GUS activity in transgenic Arabidopsis containing ACR11 promoter-GUS fusion. (A) 3-day-old, (B) 5-day-old, (C) 7day-old, (D) 10-day-old, (E) 14-day-old seedlings. (F) Flower buds and mature flowers. (G) Close-up of a mature flower. (H) A mature silique. 
they also contain multiple copies of the ACT domain. We thus adopted the term "ACT domain repeats (ACR)" and named these proteins ACR9 to ACR12, respectively.

Amino acid sequence alignment and phylogenetic analysis clearly divided these ACR proteins into three different groups. The originally identified ACR1 to ACR8 proteins contain four copies of the ACT domain and belong to Group I. The ACR9 and ACR10 proteins have three copies of the ACT domain, which are classified as Group II ACR proteins. The amino acid sequences of ACR9 and ACR10 are very similar throughout the entire polypeptides. Moreover, the gene structures of ACR 9 and ACR10 are almost identical (Figure 1B), which suggests that these two genes are recently duplicated in the Arabidopsis genome during evolution. By contrast, Group III ACR proteins, including ACR11 and ACR12, contain two copies of the ACT domain. The gene structures of $A C R 11$ and $A C R 12$ are similar. However, the encoded amino acid sequences are not conserved in the $\mathrm{N}$-terminal regions. The rest of the amino acid sequences, e.g. residues 74 to 290 of ACR11, and residues 85 to 301 of ACR12, are highly conserved. The non-conserved $\mathrm{N}$-terminal amino acid sequences of ACR11 and ACR12 are predicted to be transit peptides, which target these proteins to the chloroplast. Thus Group III ACR proteins may be localized to the chloroplast.

\section{Group III ACR proteins are localized to the chloroplast}

Most amino acids are synthesized in the chloroplast. It is expected that some regulatory proteins involved in amino acid metabolism or signaling may also exist in the chloroplast. The Arabidopsis Group III ACR proteins are good candidates in this regard, because they are predicted to target to the chloroplast. We used transient expression assay in Arabidopsis protoplasts to verify that the ACR11- and ACR12-GFP fusion proteins are localized to the chloroplast (Figure 3). After the removal of transit peptide, the mature ACR11 and ACR12 proteins are only composed of two ACT domains. It is conceivable that the ACT domains of the ACR11 and ACR12 proteins may serve as amino acid binding domains. Upon binding to specific amino acids, the ACR11 and ACR12 proteins may regulate the activities of amino acid biosynthetic enzymes in the chloroplast. Alternatively, the two ACT domains of the ACR11 and ACR12 proteins may function as specific amino acid sensors in the chloroplast, which are similar to those of bacterial GlnD proteins. It will be interesting to further characterize the functions of the Arabidopsis ACR11 and ACR12 proteins and their homologs in the other plants.

\section{ACR11 and GLN2 are in the same coexpressed gene network}

Genes involved in related biological pathways are often coordinately regulated [44]. The coexpression analysis obtained from the ATTED-II database (http://atted.jp) may help us to identify the functions of Arabidopsis ACR11 and ACR12. In the ATTED-II database, the ACR11 and ACR12 genes have distinct coexpressed gene networks (Figure 4). It is possible that the proteins encoded by these two homologous genes may also have distinct functions in Arabidopsis chloroplasts. It is intriguing that the Arabidopsis ACR11 and GLN2 are in the same coexpressed gene network. Moreover, the mutual rank for coexpression of these two genes is the highest in their respective gene networks (Figure 4). It is well known that the expression of Arabidopsis GLN2 is regulated by light and sugars [46]. We used RNA gel blot analysis to examine the effects of light and sucrose on the expression of $A C R 11$. Interestingly, the results are in accordance with the coexpression analysis in the database. Steady-state levels of both ACR11 and GLN2 mRNAs are increased by treatments of sucrose and light (Figure 6). The highly cooperative expression of ACR11 and GLN2 observed in our experiments and in the database suggests that these two genes may belong to the same functional module. The GLN2 encodes a chloroplastic GS2, which is the major enzyme for glutamine synthesis in the chloroplast. However, the functions of the chloroplast-localized ACR11 protein are completely unknown. The ACR11 and GLN2 genes have the highest coexpression relationship in the Arabidopsis genome suggests that the ACR11 protein may have functions related to GS2.

The relationship between Arabidopsis ACR11 and GS2 is reminiscent of the PII-GlnD system in the regulation of $g \ln A$ gene expression and GS enzyme activity in bacteria $[7-10,18]$. In addition to the ACR homologs in plants, the amino acid sequence of ACR11 is most similar to the ACT domains of the bacterial sensor protein GlnD (e.g. uridylyltransferase). Thus the ACR11 (At1g16880) was annotated as uridylyltransferase-related protein in the GenBank (NM_101549). The bacterial GlnD protein may sense the availability of glutamine, possibly via the two ACT domains in the C-terminal region, to regulate GS enzyme activity and its gene expression [21]. It is possible that the Arabidopsis ACR11 protein may also use its ACT domains to sense the availability of glutamine in the chloroplast, and then regulates GS2 activity or glutamine metabolism.

ACR11 and ACR12, putative amino acid sensor proteins in the chloroplast

Chloroplast is the site of active primary and secondary nitrogen assimilation inside a plant cell. The assimilation 
of ammonia into glutamine is the major pathway to convert inorganic nitrogen into organic nitrogen in plants. Thus it is expected that plants may have a mechanism to sense the availability of glutamine inside the chloroplast. In E. coli, glutamine may serve as a signaling molecule to affect the expression of nitrogen assimilatory genes and the activities of nitrogen metabolic enzymes [7]. The two ACT domains located in the $\mathrm{C}$-terminal region of the $\mathrm{GlnD}$ protein are considered as glutamine sensors in bacteria [21]. Little is known about amino acid sensing and signaling in plants. Interestingly, the ACR11 and ACR12 proteins are composed of two ACT domains, and are localized to the chloroplast. It is conceivable that the ACR11 and ACR12 proteins may function as amino acid sensors in Arabidopsis. Future studies are needed to determine the functions of these chloroplastic ACR proteins.

\section{Conclusions}

Although the ACT domains have high sequence divergence, there is a common regulatory theme among these domains. The Arabidopsis ACR proteins contain multiple copies of the ACT domain and their functions are largely unknown. In this study, we identified two new groups of ACR proteins in Arabidopsis. Group II ACR proteins, ACR9 and ACR10, have three copies of the ACT domain. Whereas group III ACR proteins, ACR11 and ACR12, contain two copies of the ACT domain, and are localized to the chloroplast. The activities of $A C R 11$ promoter-GUS are mainly detected in mature leaves. Moreover, the expression of ACR11 and GLN2 is highly coordinated. The ACR11 may function as a regulatory protein involved in glutamine metabolism or sensing in Arabidopsis.

\section{Methods}

\section{Plant material and growth conditions}

Arabidopsis thaliana ecotype Columbia-0 was grown in soils in the greenhouse on a 16-h light/8-h dark cycle at $23^{\circ} \mathrm{C}$. Roots, leaves, stems, flowers, and siliques from the same batch of 6-week-old soil-grown plants were used for total RNA extraction. For experiments in which plants were transferred to $0 \%, 3 \%$ sucrose or $3 \%$ mannitol, seeds were sown on $1.5 \mathrm{~cm} \times 8 \mathrm{~cm}$ Nylon nets with $250 \mu \mathrm{m}$ mesh size (Tetko, Elmsford, NY, USA, catalog no. 3-250/50), placed on the surface of the Murashige and Skoog (MS) plates [MS salts (Sigma-Aldrich Co., St. Louis, MO), $\mathrm{pH}$ adjusted to 5.7 with $1 \mathrm{~N} \mathrm{KOH}, 0.8 \%$ (w/ v) phytoagar] containing $3 \%$ sucrose. After cold treatment at $4^{\circ} \mathrm{C}$ for $48 \mathrm{~h}$, plates were vertically placed in a $23^{\circ} \mathrm{C}$ chamber on a 16 -h light $/ 8$-h dark cycle for two weeks. The plants and the nylon nets were lifted and transferred to fresh MS media containing 0\%, 3\% sucrose or $3 \%$ mannitol, and dark-adapted or grown in continuous light for $48 \mathrm{~h}$.

\section{Cloning of Arabidopsis ACR9, ACR10, ACR11 and ACR12 cDNAs}

Total RNA from 2-week-old Arabidopsis was used for reverse transcription-PCR (SuperScript II RT Kit, Invitrogen, Carlsbad, CA) to amplify ACR9 (At2g39570), ACR10 (At2g36840), ACR11 (At1g16880) and ACR12 (At5g04740) cDNAs. The following primers were used to amplify full-length cDNAs: $A C R 9,5$ '-TGTTGTT GATTCATTGGCTC-3' and 5'-AGTAGTAGATGAATATATTG-3'; ACR10, 5'-ATAGGAGGAACAACACAAAC-3' and 5'-TTACTATGAAACCCACACAG-3'; ACR11, 5'-AAAAGGATCCATGGCTATGGCCTCT GCTTC-3' and 5'-GGGGAGGCCTGAAACTTGACTC GTCAGTTG-3'; ACR12, 5'-AGGGACCGGTATGGCG TTCTCGAGTTCCAT-3' and 5'-GGGGACCGGTGTAGCTGTCAATGTCAGTTT-3'. The PCR products were cloned into pGEM-T easy vector (Promega Co., Madison, WI) and provided for sequencing. The Arabidopsis $A C R 9$ to $A C R 12$ cDNA sequences were verified and deposited in the GenBank (JF797174 to JF797177).

\section{Sequence analysis}

The amino acid sequences of Arabidopsis ACR1 (NM_125986), ACR2 (NM_122441), ACR3 (NM_179566), ACR4 (NM_202378), ACR5 (NM_126420), ACR6 (NM_111065), ACR7 (NM_118407), ACR8 (NM_101114), ACR9 (JF797174), ACR10 (JF797175), ACR11 (JF797176), ACR12 (JF797177), and amino acid residues 708-890 of E. coli GlnD (M96431) were aligned by ClustalW2 with default settings (http://www.ebi.ac.uk/Tools/msa/clustalw2/). The neighbor-joining algorithm was used to obtain the phylogenetic tree. The sequence alignment was shaded with BOXSHADE 3.21 (http://www.ch.embnet.org/software/BOX_form.html). InterProScan (http://www.ebi.ac. $\mathrm{uk} / \mathrm{Tools} / \mathrm{pfa} / \mathrm{iprscan} /$ ) was used to analyze the domain composition of ACR9 to ACR12. PSORT (http://www. psort.org/) and TargetP (http://www.cbs.dtu.dk/services/ TargetP/) were used to predict the subcellular localization of ACR9 to ACR12. ChloroP (http://www.cbs.dtu. $\mathrm{dk} /$ services/ChloroP/) was used to predict the transit peptide cleavage sites of ACR11 and ACR12. The $A C R 11$ and ACR12 coexpression gene networks were obtained from the ATTED-II database (http://atted.jp/).

\section{ACR11- and ACR12-GFP fusion constructs}

The GFP expression vector pHBT, designed for transient expression assays [47], was used to construct the ACR11- and ACR12-GFP fusions. A BamHI/StuI fragment from the pGEM-T-ACR11 clone containing the full-length $A C R 11 \mathrm{cDNA}$ was subcloned into the pHBT vector to create an ACR11-GFP fusion construct. The $\mathrm{N}$-terminal cDNA sequence encoding the first 94 amino acids of ACR12 was amplified by PCR using primers 5'- 
GGAAGGATCCATGGCGTTCTCGAGTTCCATC-3' and 5'-GGAAAGGCCTCATTGGAACAACGTCGTCATC-3'. The PCR product was digested with BamHI and $S t u \mathrm{I}$, and cloned into the N-terminus of the GFP in the pHBT vector. The resulting construct, ACR12-GFP, contains the putative transit peptide of ACR12 fused to a GFP. The obtained ACR11- and ACR12-GFP constructs, and the GFP empty vector were transformed into Arabidopsis protoplasts using polyethylene glycol (PEG)-mediated transient gene expression [47] and observed under confocal laser scanning microscope (510 META Zeiss) $16 \mathrm{~h}$ after transformation.

\section{RNA gel blot analysis}

Arabidopsis total RNA was isolated using a phenol extraction protocol [48]. Total RNA $(10 \mu \mathrm{g})$ was separated in standard formaldehyde gel by electrophoresis and blotted onto a nylon membrane. For detection of ACR11 and GLN2 mRNA, digoxigenin (DIG)-labeled single-stranded DNA probes were generated by PCR using the following primers: ACR11 (At1g16880), 5'-ATGGCTATGGCCT CTGCTTC-3', 5'-GAAACTTGACTCGTCAGTTG-3'; GLN2 (At5g35630), 5'-GGTGAAGTTATGCCTGGA-3', 5'-GAGAGACCACATAGACAC-3'. DIG probe labeling, pre-hybridization, hybridization, wash conditions and detection were performed according to the BoehringerMannheim Genius System User's Guide: DIG Application Manual for Filter Hybridization.

\section{ACR11 promoter-GUS fusion}

ACR11 (At1g16880) and its upstream gene At1g16870 are in an opposite orientation. There are 638 nucleotides between the initiation codons (ATG) of these two genes. The putative promoter of $A C R 11$ ( -1 to -625 of the start codon) was amplified from the Arabidopsis genomic DNA by PCR using the primers 5'-CACCTCTAGACACTCAAAAATCGGAATTAA-3' and 5'-AACAAAG CTTATCTCTTGAGTCTGACTCAA-3'. The PCR product was cloned into the pCR2.1-TOPO vector (TOPO TA Cloning Kit, Invitrogen) and the sequence was confirmed. A HindIII/XbaI fragment containing the $0.625 \mathrm{~kb}$ $A C R 11$ promoter region was subcloned into the pBI101 binary vector to create an $A C R 11$ promoter-GUS fusion construct that was transformed into the Agrobacterium tumefaciens strain GV3101.

The floral dip method was used for Arabidopsis transformation [49]. Several independent ACR11 promoterGUS Arabidopsis transgenic lines were grown to T3 homozygous and stained for GUS activity [50].

\section{Acknowledgements}

We thank Mei-Jane Fang for assistance in confocal microscopy. This work was supported by grants to MHH from National Science Council (NSC 992311-B-001-007-MY3) and Academia Sinica (98-CDA-L04) of Taiwan.

\section{Authors' contributions}

TYS carried out protoplast transient assays. TYC carried out RNA blot analysis, TYS, TYC and CPH participated in molecular cloning and promoter-GUS analysis. MHH conceived the study, carried out bioinformatic analysis and sequence alignment, and wrote the manuscript. All authors read and approved the final manuscript.

Received: 24 May 2011 Accepted: 24 August 2011

Published: 24 August 2011

\section{References}

1. Forde BG, Lea PJ: Glutamate in plants: metabolism, regulation, and signalling. J Exp Bot 2007, 58:2339-2358.

2. Lam HM, Chiu J, Hsieh MH, Meisel L, Oliveira IC, Shin M, Coruzzi G: Glutamate-receptor genes in plants. Nature 1998, 396:125-126.

3. Coruzzi G, Bush DR: Nitrogen and carbon nutrient and metabolite signaling in plants. Plant Physiol 2001, 125:61-64.

4. Walch-Liu P, Liu LH, Remans T, Tester M, Forde BG: Evidence that Lglutamate can act as an exogenous signal to modulate root growth and branching in Arabidopsis thaliana. Plant Cell Physiol 2006, 47:1045-1057.

5. Tabuchi M, Abiko T, Yamaya T: Assimilation of ammonium ions and reutilization of nitrogen in rice (Oryza sativa L.). J Exp Bot 2007, 58:2319-2327.

6. Forde $B G$, Walch-Liu P: Nitrate and glutamate as environmental cues for behavioural responses in plant roots. Plant Cell Environ 2009, 32:682-693.

7. Leigh JA, Dodsworth JA: Nitrogen regulation in bacteria and archaea. Annu Rev Microbiol 2007, 61:349-377.

8. Bueno R, Pahel $G$, Magasanik B: Role of $g \ln B$ and $g \ln D$ gene products in regulation of the gInALG operon of Escherichia coli. J Bacteriol 1985, 164:816-822

9. Magasanik B: Reversible phosphorylation of an enhancer binding protein regulates the transcription of bacterial nitrogen utilization genes. Trends Biochem Sci 1988, 13:475-479.

10. Ninfa AJ, Atkinson MR: PII signal transduction proteins. Trends Microbiol 2000, 8:172-179.

11. Brown MS, Segal A, Stadtman ER: Modulation of glutamine synthetase adenylylation and deadenylylation is mediated by metabolic transformation of the PII -regulatory protein. Proc Natl Acad Sci USA 1971, 68:2949-2953.

12. Garcia E, Rhee SG: Cascade control of Escherichia coli glutamine synthetase. Purification and properties of PII uridylyltransferase and uridylyl-removing enzyme. J Biol Chem 1983, 258:2246-2253.

13. Son HS, Rhee SG: Cascade control of Escherichia coli glutamine synthetase. Purification and properties of PII protein and nucleotide sequence of its structural gene. J Biol Chem 1987, 262:8690-8695.

14. McFarland N, McCarter L, Artz S, Kustu S: Nitrogen regulatory locus "glnR" of enteric bacteria is composed of cistrons ntrB and ntrC: identification of their protein products. Proc Natl Acad Sci USA 1981, 78:2135-2139.

15. Ninfa AJ, Magasanik B: Covalent modification of the $g / n G$ product, NRI, by the $g I n L$ product, NRII, regulates the transcription of the $\operatorname{g} I n A L G$ operon in Escherichia coli. Proc Natl Acad Sci USA 1986, 83:5909-5913.

16. Reitzer $\amalg$, Magasanik $B$ : Transcription of $g / n A$ in $E$. coli is stimulated by activator bound to sites far from the promoter. Cell 1986, 45:785-792.

17. Keener J, Kustu S: Protein kinase and phosphoprotein phosphatase activities of nitrogen regulatory proteins NTRB and NTRC of enteric bacteria: roles of the conserved amino-terminal domain of NTRC. Proc Natl Acad Sci USA 1988, 85:4976-4980.

18. Magasanik $B$ : The regulation of nitrogen utilization in enteric bacteria. $J$ Cell Biochem 1993, 51:34-40.

19. Atkinson MR, Kamberov ES, Weiss RL, Ninfa AJ: Reversible uridylylation of the Escherichia coli PII signal transduction protein regulates its ability to stimulate the dephosphorylation of the transcription factor nitrogen regulator I (NRI or NtrC). J Biol Chem 1994, 269:28288-28293.

20. Jiang P, Ninfa AJ: Escherichia coli Pll signal transduction protein controlling nitrogen assimilation acts as a sensor of adenylate energy charge in vitro. Biochemistry 2007, 46:12979-12996.

21. Zhang Y, Pohlmann EL, Serate J, Conrad MC, Roberts GP: Mutagenesis and functional characterization of the four domains of $\mathrm{G} \ln \mathrm{D}$, a bifunctional nitrogen sensor protein. J Bacteriol 2010, 192:2711-2721.

22. Hsieh MH, Lam HM, van de Loo FJ, Coruzzi G: A PII-like protein in Arabidopsis: putative role in nitrogen sensing. Proc Natl Acad Sci USA 1998, 95:13965-13970. 
23. Sugiyama K, Hayakawa T, Kudo T, Ito T, Yamaya T: Interaction of Nacetylglutamate kinase with a PII-like protein in rice. Plant Cell Physiol 2004, 45:1768-1778.

24. Schuller DJ, Grant GA, Banaszak LJ: The allosteric ligand site in the Vmaxtype cooperative enzyme phosphoglycerate dehydrogenase. Nat Struct Biol 1995, 2:69-76.

25. Aravind L, Koonin EV: Gleaning non-trivial structural, functional and evolutionary information about proteins by iterative database searches. J Mol Biol 1999, 287:1023-1040.

26. Chipman DM, Shaanan B: The ACT domain family. Curr Opin Struct Biol 2001, 11:694-700.

27. Liberles JS, Thorolfsson M, Martinez A: Allosteric mechanisms in ACT domain containing enzymes involved in amino acid metabolism. Amino Acids 2005, 28:1-12.

28. Grant GA: The ACT domain: a small molecule binding domain and its role as a common regulatory element. J Biol Chem 2006, 281:33825-33829.

29. Grant $G A, H u Z, X u X L$ : Specific interactions at the regulatory domainsubstrate binding domain interface influence the cooperativity of inhibition and effector binding in Escherichia coli D-3-phosphoglycerate dehydrogenase. J Biol Chem 2001, 276:1078-1083.

30. Grant GA, Kim SJ, Xu XL, Hu Z: The contribution of adjacent subunits to the active sites of D-3-phosphoglycerate dehydrogenase. J Biol Chem 1999, 274:5357-5361.

31. Mendel S, Elkayam T, Sella C, Vinogradov V, Vyazmensky M, Chipman DM, Barak Z: Acetohydroxyacid synthase: a proposed structure for regulatory subunits supported by evidence from mutagenesis. J Mol Biol 2001, 307:465-477.

32. Chinchilla D, Schwarz FP, Eisenstein E: Amino acid substitutions in the Cterminal regulatory domain disrupt allosteric effector binding to biosynthetic threonine deaminase from Escherichia coli. J Biol Chem 1998, 273:23219-23224.

33. Gallagher DT, Gilliland GL, Xiao G, Zondlo J, Fisher KE, Chinchilla D, Eisenstein E: Structure and control of pyridoxal phosphate dependent allosteric threonine deaminase. Structure 1998, 6:465-475.

34. Siltberg-Liberles J, Martinez A: Searching distant homologs of the regulatory ACT domain in phenylalanine hydroxylase. Amino Acids 2009, 36:235-249.

35. Wilson TJ, Argaet VP, Howlett GJ, Davidson BE: Evidence for two aromatic amino acid-binding sites, one ATP-dependent and the other ATPindependent, in the Escherichia coli regulatory protein TyrR. Mol Microbiol 1995, 17:483-492.

36. Ettema TJ, Brinkman AB, Tani TH, Rafferty JB, Van Der Oost J: A novel ligand-binding domain involved in regulation of amino acid metabolism in prokaryotes. J Biol Chem 2002, 277:37464-37468.

37. Schreiter ER, Sintchak MD, Guo Y, Chivers PT, Sauer RT, Drennan CL: Crystal structure of the nickel-responsive transcription factor NikR. Nat Struct Biol 2003, 10:794-799.

38. Devedjiev Y, Surendranath Y, Derewenda U, Gabrys A, Cooper DR, Zhang RG, Lezondra L, Joachimiak A, Derewenda ZS: The structure and ligand binding properties of the $B$. subtilis $Y k o F$ gene product, a member of a novel family of thiamin/HMP-binding proteins. J Mol Biol 2004, 343:395-406.

39. Feller A, Hernandez JM, Grotewold E: An ACT-like domain participates in the dimerization of several plant basic-helix-loop-helix transcription factors. J Biol Chem 2006, 281:28964-28974.

40. Hsieh MH, Goodman HM: Molecular characterization of a novel gene family encoding ACT domain repeat proteins in Arabidopsis. Plant Physiol 2002, 130:1797-1806

41. Hayakawa T, Kudo T, Ito T, Takahashi N, Yamaya T: ACT domain repeat protein 7, ACR7, interacts with a chaperone HSP18.0-CII in rice nuclei. Plant Cell Physiol 2006, 47:891-904.

42. Liu Q: Computational identification and systematic analysis of the ACR gene family in Oryza sativa. J Plant Physiol 2006, 163:445-451.

43. Kudo T, Kawai A, Yamaya T, Hayakawa T: Cellular distribution of ACT domain repeat protein 9, a nuclear localizing protein, in rice (Oryza sativa). Physiol Plant 2008, 133:167-179.

44. Eisen MB, Spellman PT, Brown PO, Botstein D: Cluster analysis and display of genome-wide expression patterns. Proc Natl Acad Sci USA 1998, 95:14863-14868.

45. Obayashi T, Nishida K, Kasahara K, Kinoshita K: ATTED-II updates: conditionspecific gene coexpression to extend coexpression analyses and applications to a broad range of flowering plants. Plant Cell Physiol 2011, 52:213-219.

46. Oliveira IC, Coruzzi GM: Carbon and amino acids reciprocally modulate the expression of glutamine synthetase in Arabidopsis. Plant Physiol 1999, 121:301-310.

47. Chiu W, Niwa Y, Zeng W, Hirano T, Kobayashi H, Sheen J: Engineered GFP as a vital reporter in plants. Curr Biol 1996, 6:325-330.

48. Jackson $\mathrm{AO}$, Larkins $\mathrm{BA}$ : Influence of ionic strength, $\mathrm{pH}$, and chelation of divalent metals on isolation of polyribosomes from tobacco leaves. Plant Physiol 1976, 57:5-10.

49. Clough SJ, Bent AF: Floral dip: a simplified method for Agrobacteriummediated transformation of Arabidopsis thaliana. Plant J 1998, 16:735-743.

50. Jefferson RA, Kavanagh TA, Bevan MW: GUS fusions: beta-glucuronidase as a sensitive and versatile gene fusion marker in higher plants. Embo $J$ 1987, 6:3901-3907.

doi:10.1186/1471-2229-11-118

Cite this article as: Sung et al:: The ACR11 encodes a novel type of chloroplastic ACT domain repeat protein that is coordinately expressed with GLN2 in Arabidopsis. BMC Plant Biology 2011 11:118.

\section{Submit your next manuscript to BioMed Central and take full advantage of:}

- Convenient online submission

- Thorough peer review

- No space constraints or color figure charges

- Immediate publication on acceptance

- Inclusion in PubMed, CAS, Scopus and Google Scholar

- Research which is freely available for redistribution
C Biomed Central 